\title{
Laboratory technique for quantitative thermal emissivity measurements of geological samples
}

\author{
George Mathew $^{1, *}$, Archana Nair ${ }^{1}$, T K Gundu RaO ${ }^{2}$ and Kanchan Pande ${ }^{1}$ \\ ${ }^{1}$ Department of Earth Sciences, Indian Institute of Technology, Bombay, Powai, Mumbai 400 076, India. \\ ${ }^{2}$ Sophisticated Analytical Instrumentation Centre, Indian Institute of Technology, Bombay, \\ Powai, Mumbai 400 076, India. \\ *e-mail: gmathew@iitb.ac.in
}

Thermal infrared spectroscopy is a powerful technique for the compositional analysis of geological materials. The spectral feature in the mid-IR region is diagnostic of the mineralogy and spectral signatures of mixtures of minerals that add linearly, and therefore, can be used as an important tool to determine the mineralogy of rocks in the laboratory and remotely for planetary exploration. The greatest challenge in the emission measurement lies in the measurement of the weak thermal photons emitted from geological materials in a laboratory setup, and accurately records the temperature of the rock sample. The present work pertains to the details of a new Thermal Emission Spectrometer (TES) laboratory that has been developed under the ISRO Planetary Science and Exploration (PLANEX) programme, for emission related mineralogical investigations of planetary surfaces. The focus of the paper is on the acquisition and calibration technique for obtaining emissivity, and the deconvolution procedure to obtain the modal abundances of the thermal emission spectra in the range of 6-25 $\mu \mathrm{m}$ using Fourier Transform Infrared (FTIR) spectroscopy. The basic technique is adopted from the work of Ruff et al (1997). This laboratory at the Department of Earth Sciences, IIT-Bombay is currently developing pure end mineral library of mineral particulates $(<65 \mu \mathrm{m})$, and adding new end members to the existing ASU spectral library. The paper argues the need for considering Lunar Orbiter Thermal Emission Spectrometer (LOTES) for future Indian Moon mission programme (Chandrayan-II) to determine evidences of varied lithologies on the lunar surface.

\section{Introduction}

Vibrational spectroscopy provides an important tool for studying geological samples both in the laboratory and for remote sensing applications. Infrared (IR) spectroscopy is a non-destructive tool for examining rocks and minerals for providing valuable knowledge about the chemical forces between atoms, and vibrational frequencies found in molecules (Clark 2004). The various physical processes that give rise to IR spectra in minerals are now well understood and documented (Farmer 1974; Hawthorne 1988; King et al 2004). There exits several methods for performing vibrational spectroscopy, such as transmission, reflection, emission and Raman methods; however, for compositional mapping of terrestrial or extraterrestrial bodies reflection techniques in the Visible NearInfrared (VIS-NIR) $(400-2500 \mathrm{~nm})$ and emission method in the mid-IR region are the most useful techniques to derive useful geological information.

Emission of radiation from a sample occurs due to thermal vibration of its molecules. The spectral features arising from the fundamental, combination, and overtone vibrational bands, appear as troughs or emissivity lows in an emission spectra. The thermal infrared photons are measured usually between the wavelength regions of $8-50 \mu \mathrm{m}$

Keywords. Emissivity; infrared spectroscopy; thermal emission; planetary geology. 
or $1200-200 \mathrm{~cm}^{-1}$ in a typical laboratory setup (Ruff et al 1997). Spectral emissivity can also be determined from reflectance using Kirchhoff's law, $\varepsilon=1-R$ (Nicodemus 1965; Salisbury et al 1994) where $\varepsilon$ is emissivity and $R$ is the reflectance. This is more valid for reflectance measurement taken using hemispherical attachment. However, this technique is not feasible for extraterrestrial exploration as Sun's reflected energy does not cover this region. The Kirchhoff's law can be qualitatively applied in remote sensing using bidirectional reflectance using biconical reflectance attachment (Salisbury et al 1993).

The fundamental difference between the reflectance and emission technique is the source of the infrared energy. Typically, the most practical method for emission analysis is to heat the sample to a higher temperature. Although an emission signal is not restricted to materials heated to high temperatures, the sample signal is greater at higher temperature, and is easier to differentiate from the noise produced by an instrument and environment. The collection of emission spectra by definition is hemispherically integrated radiance, and therefore, eliminates most of the angular dependencies that results from a reflectance spectroscopy, and moreover, collection of spectra in the emission mode more accurately mimics the emission from natural surfaces observed by remote sensing instruments (Ramsey 2004). The other advantage is that the emission of infrared radiance is what naturally occurs on the Earth's surface. Therefore, laboratory based measurements are directly comparable to remote sensing data. The average surface temperature of the Earth $\left(\sim 27^{\circ} \mathrm{C}\right.$ or $\left.300 \mathrm{~K}\right)$ produces a maximum emitted flux at $9.7 \mu \mathrm{m}$, whereas at wavelengths longer than $\sim 5 \mu \mathrm{m}$, the solar reflected energy approaches zero, hence, no reflected IR energy is observed at values greater than $5 \mu \mathrm{m}$. The reflectance spectral studies hence becomes nonfeasible, however, emission spectra can still be recorded for energies $>5 \mu \mathrm{m}$ either in the field, air or in space-based mapping.

In the thermal-IR region, two important windows occur at 8.0-9.2 $\mu \mathrm{m}$ and $10.2-12.4 \mu \mathrm{m}$, which are separated by an absorption band due to ozone present in the upper atmosphere. For remote sensing from aerial platforms, the thermal channel between 8 and $14 \mu \mathrm{m}$ is usually used. The fundamental frequencies of geological materials lie in the wavelengths above $6 \mu \mathrm{m}$, providing the most diagnostic information for the identification of almost all minerals (Walter and Salisbury 1989; Salisbury et al 1993; Salisbury 1993). The greatest challenge in the emission measurement lies in the measurement of the weak thermal photons emitted from geological materials in a laboratory setup, and to accurately record the temperature of the rock sample. Currently three laboratories in US have developed the facility to measure thermal infrared (TIR) spectrum of geological samples. The experimental setup developed for the present study has followed the Ruff et al (1997) technique. The other recently developed laboratory is at the Berlin Institute of Planetary Research, German Aerospace Centre for the MERTIS and Venus Express programme (Maturilli et al 2008).

In recent years interest in emission spectroscopy of planetary surfaces has been greater. It was included both on orbiters and rovers for studying Mars. Today, most missions on Mars have onboard a TES instrument dedicated to emission spectral measurements: TES on Mars Global Surveyor (Christensen et al 2001) and imaging spectrometer THEMIS on Mars Odyssey (Christensen et al 2003). The two Mars Exploration Rovers (MER) are still providing a wealth of high spectral resolution data. The most recent data includes the finding of hydrothermal silica rich deposits in association with volcanic materials (Squyres et al 2008): The Planetary Fourier Spectrometer (PFS) onboard Mars Express (Formisano et al 2005). The Mercury Thermal Infrared Imaging Spectrometer (MERTIS) on the ESA Bepi-Colombo mission to Mercury will map the planet with a spatial resolution of $500 \mathrm{~m}$ and a spectral resolution of $90 \mathrm{~m}$ between 7 and $14 \mu \mathrm{m}$ (Helbert et al 2005). An updated version of the MERTIS instrument is currently proposed as Selenological Radiometer and Thermal Infrared Imaging Spectrometer (SERTIS) for a German Lunar Mission (Maturilli et al 2008). The Advanced Spaceborne Thermal Emission Reflection Radiomter (ASTER) launched in 1999 is the only earth orbiter that provided five band thermal infrared data of the earth surface (Gillespie et al 1998). The active missions are currently providing wealth of compositional informations on Mars, while planned missions in the near future will be generating wealth of mid-IR emission information from various planetary bodies. The spectral data contains immense information related to the mineralogical composition, surface properties, alteration, atmospheric dust, etc. To derive reliable information from these remote sensing spectral data, a greater understanding of similar analogue materials using laboratory spectroscopic measurements is required. The information related to wavelength positions, spectral contrasts, the various troughs and peaks of various minerals, mixtures and grain sizes are required for the correct identification of planetary materials.

The focus of the present work is on the instrumentation setup, measurement procedures for geological analogues and calibration techniques to 


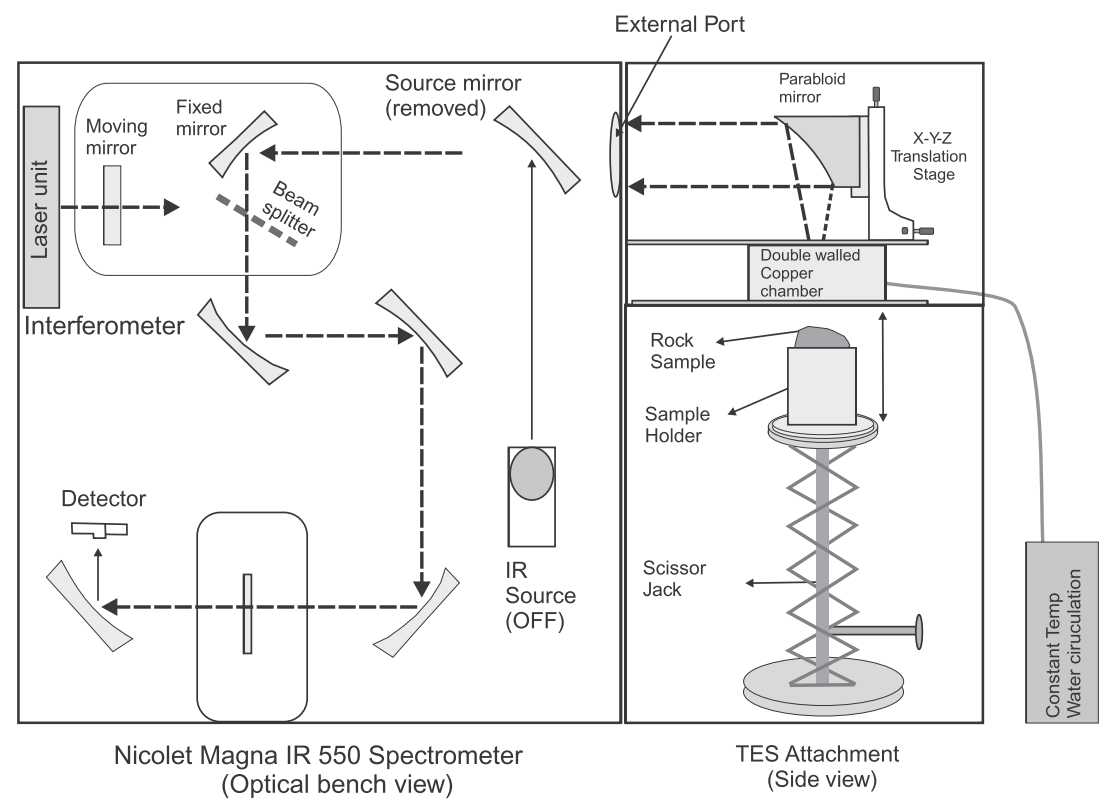

Figure 1. Diagram showing the FTIR optical bench layout and the thermal emission attachment apparatus.

obtain the emissivity. A thermal emission spectrometer (TES) laboratory at the Department of Earth Science, IIT-Bombay has been developed under ISRO-PLANEX (Planetary Sciences and Exploration) programme. The present work also discusses the deconvolution technique and, finally argues the need for Lunar Orbiter Thermal Emission Spectrometer (LOTES) for lunar surface mapping.

\section{Thermal emission spectrometer (TES) instrumentation}

The present setup uses a Fourier Transform Infrared (FTIR) spectrometer, which is modified for the acquisition of thermal emission spectra by removing its external port and attaching the thermal emission sample apparatus to it. The IR source in the spectrometer is kept off. A Fourier Transform Infrared (FTIR) spectrometer using Michelson interferometer technique is adapted for emission spectroscopy by removing the mirror closest to the emission port and by disconnecting the internal source (figure 1). The sample itself is used as the infrared source by directing the optical path through the external port to the sample chamber using a parabolic mirror. The sample apparatus is attached to the FTIR external port to direct the energy from the sample source to the detector (figure 2). The energy from the sample source is acquired using a Nicolet Magna 550 FTIR spectrometer equipped with an uncooled Deuterated Triglycine Sulphate (DTGS) detector and $\mathrm{KBr}$ beam splitter. The spectra were obtained at
$4 \mathrm{~cm}^{-1}$ sampling over $1600-400 \mathrm{~cm}^{-1}(\approx 6-25 \mu \mathrm{m})$. The basic design of the thermal emission accessory is adapted from Ruff et al (1997).

The thermal emission accessory consists of two glove boxes. The upper one consists of the emission optics mounted on the double walled copper chamber, and the lower is the sample chamber (figure 2). The emission optics consists of a parabolic mirror that collects the energy from the sample and directs it through the external port towards the interferometer. The $7.5 \mathrm{~cm}$ diameter off axis parabolic mirror, with a focal length of $11.9 \mathrm{~cm}$ is used to produce a $37^{\circ}$ cone normal to the sample surface and elliptical field of view. The use of a parabolic mirror increases the signal strength by allowing the collection of energy over a larger solid angle (Ruff et al 1997). The mirror is mounted coaxially with the sample chamber on an X-Y-Z translation stage. The translation stage is used for the precise alignment of the mirror. It is used to adjust the parabolic mirror to receive maximum energy from the sample source and direct it towards the interferometer. The copper chamber is designed to avoid the heat loss from the sample, once the sample is jacked inside. The chamber is a double walled copper chamber of $20 \mathrm{~cm}$ diameter closed at the top, except for a $1.5 \mathrm{~cm}$ opening through which the emitted photons exit. To avoid undue heating and radiation of the chamber, water is circulated to maintain a constant temperature at $23.0^{\circ} \mathrm{C} \pm 0.03$. The interior surfaces have been painted with Krylon ultra flat black 1601 paint that has an emissivity $\geq 0.95$. The bottom of the chamber is open to allow samples to be raised inside the cavity. This chamber acts as a black body cavity 


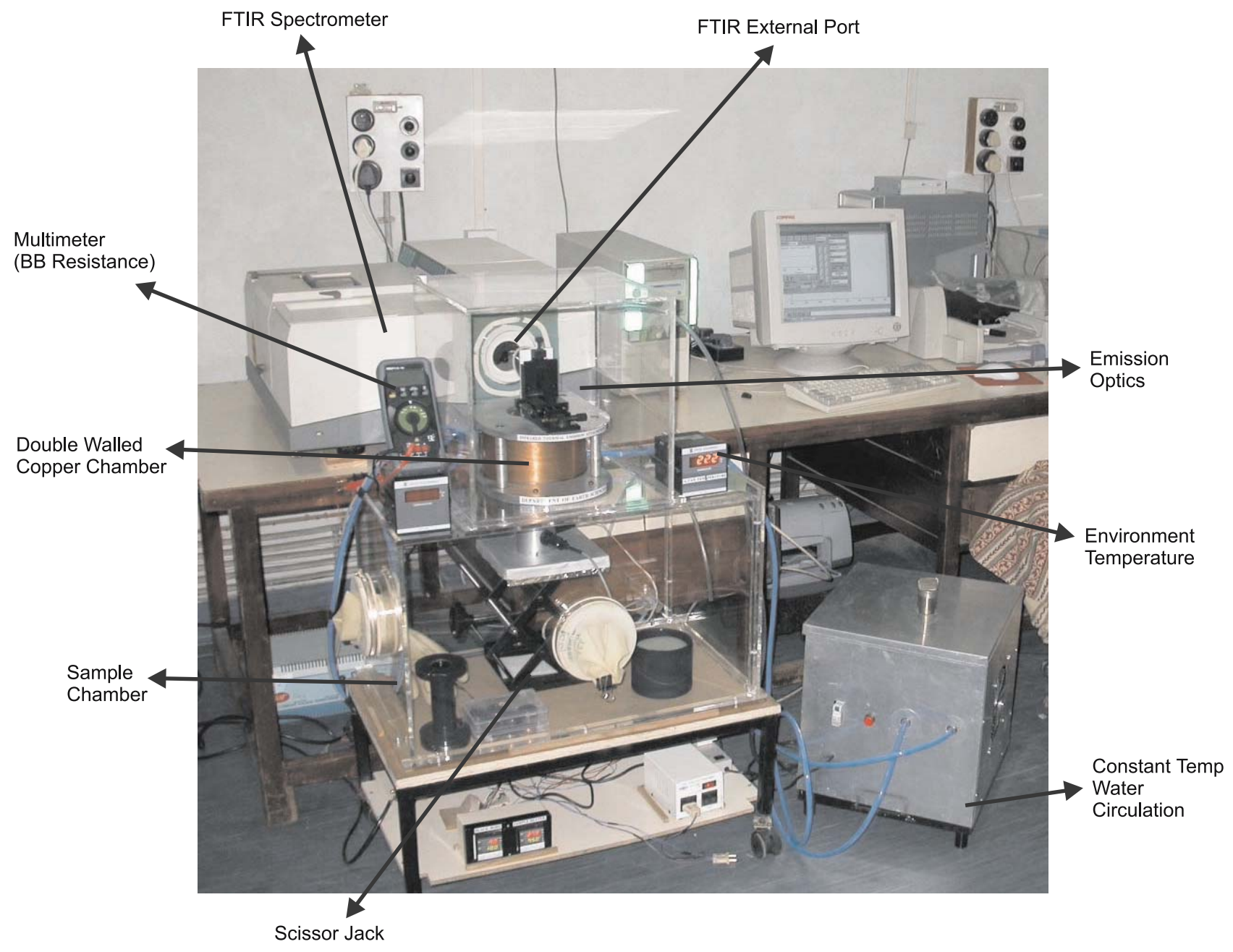

Figure 2. The thermal emission spectrometer (TES) laboratory setup at IIT-Bombay.

allowing only the sample energy and the reflected energy to the detector. A thermocouple attached to the inner wall is used to record environment temperature (figure 2). To reduce the effects of variable atmospheric conditions in the laboratory, the spectrometer and glove box are purged continuously with ultra-pure $\mathrm{N}_{2}$ gas. This also avoids exposure of the $\mathrm{KBr}$ beam splitter and lenses to moisture.

\section{Measurement}

The measurement procedure for acquiring the spectra of geological samples (rock/particulates) begins by first measuring the two blackbody temperatures at $100^{\circ} \mathrm{C}$ (hot) and $70^{\circ} \mathrm{C}$ (warm). This gives the instrument response function. The second step involves measurement of the sample radiance. For this usually fist size rock samples are heated in an oven at $80^{\circ} \mathrm{C}$ for about $12 \mathrm{~h}$ in order to increase the signal-to-noise ratio. The hot samples are then immediately jacked into the copper chamber.

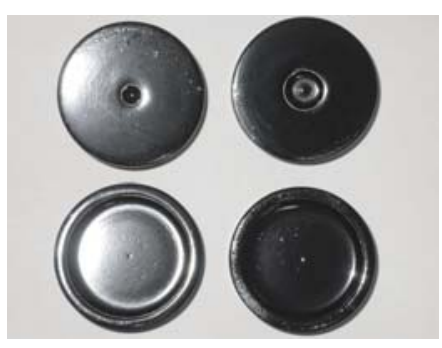

(a)

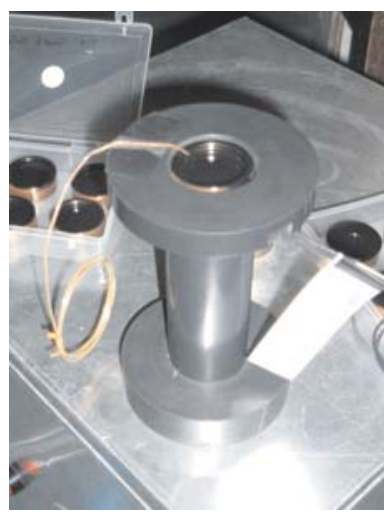

(b)
Figure 3. The accessory for measuring thermal infrared emission spectra of particulate samples. (a) Various size copper cups coated with black paint and (b) the sample holder for heating copper cups.

The spectrum is acquired for 180 interferograms during the $3^{1 / 2}$ minutes acquisition period and averaged together to produce the final spectrum. The powder samples are heated using copper cups 

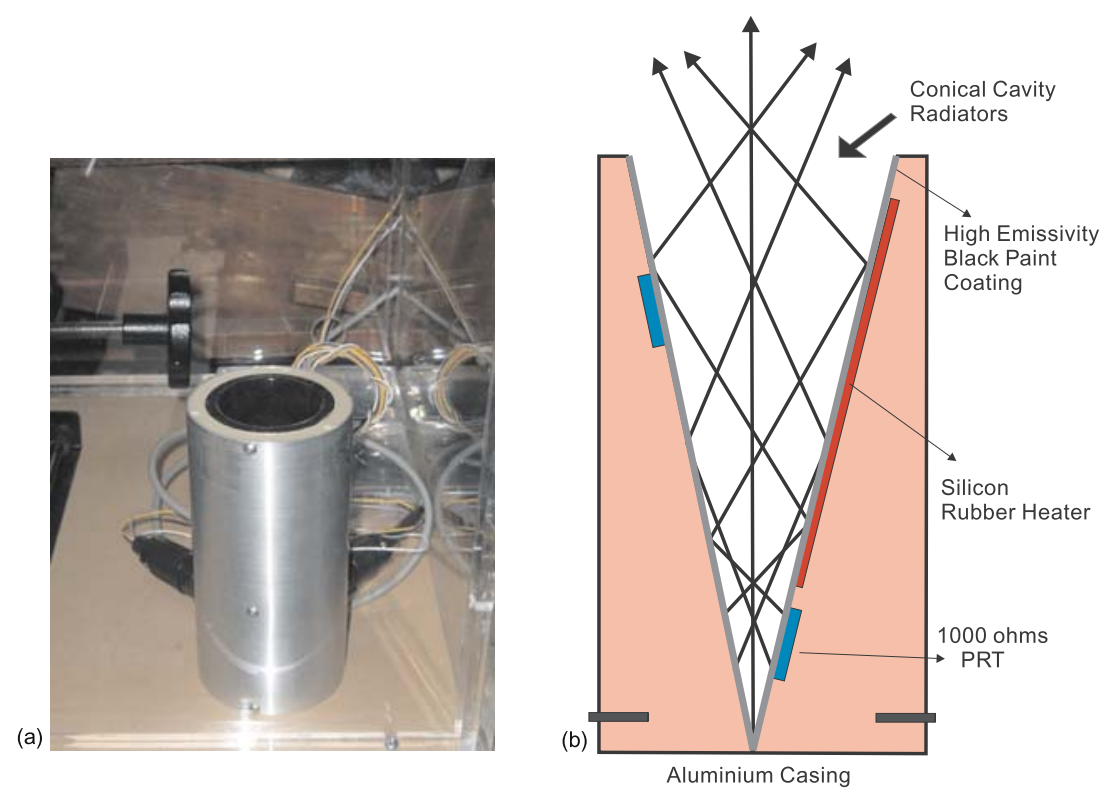

Figure 4. Blackbody target required for the instrument and spectral calibration. (a) Photo of the aluminum blackbody cylinder and (b) show the cross section diagram of the blackbody. The $30^{\circ}$ cone is jacked by silicone rubber heater, and the resistance is measured by two PRT's.

(figure 3a). Different dimension copper cups are used depending on the sample volume. The copper cups are coated with Krylon black paint on its upper exposed surface. During measurements, sample is placed on a heater unit and is maintained at $80^{\circ} \mathrm{C}$. Particulate samples in sample cups are placed in a holder that contains a heater unit. The heater unit regulates its temperature by a timeproportional temperature controller which is connected to a thermocouple. The thermocouple is so placed that it is in constant contact with the sample at its outer edge (figure $3 \mathrm{~b}$ ). Thus the set temperature and the original temperature of the sample are known. The holder is then jacked into the copper chamber for measurements.

\section{Calibration}

The calibration of the raw spectrum to emissivity is achieved following the one temperature method of Ruff et al (1997) using a single calibrated blackbody. Spectra are obtained from blackbody at two temperatures, warm $70^{\circ} \mathrm{C}$ and hot $100^{\circ} \mathrm{C}$ at a higher 250 interferogram scan. Measurement of blackbody is repeated for every batch of samples. The integral part of emission spectroscopy is thus the use of a blackbody as reference target, whose temperature can be controlled and known accurately. The blackbody used in the present study was donated to us by Prof. Phill Christensen and Dr. Steve Ruff of Mars Space Flight Facility, Arizona State University. The blackbody is a solid aluminum and cylindrical in shape with a conical cavity having an angle of $30^{\circ}$. The inside cone is coated with black high quality epoxy resin paint. The cone is jacketed from outside by silicon heater. The temperature is monitored by two $1000 \Omega$ PRT's placed one at the base and the other on the side of cone. The output of the blackbody heating unit is regulated using a time proportional temperature controllers (figure 4).

The emissivity of any material is an intrinsic property and is not dependent on sample temperature, spectrometer and environment temperature (Ruff et al 1997). Since emissivity cannot be directly measured, it is inferred from the measured radiance. Therefore, a calibration technique is used to convert the recorded emittance of a material to its emissivity (figure 5a). The emissivity is defined as the ratio of emittance of a sample $\left(\varepsilon_{\text {sample }}\right)$ to that of a blackbody $\left(\varepsilon_{\text {blackbody }}\right)$ at the same temperature $(T)$ (figure $5 \mathrm{a}$ and $\mathrm{b}$ ).

$$
\text { Emissivity, } \varepsilon=\frac{\varepsilon_{\text {sample }}(T)}{\varepsilon_{\text {blackbody }}(T)} \text {. }
$$

Thermal emission calibration software has been developed to generate the emissivity of measured samples (figure 6). Emitted radiance is the quantity that is used to infer emissivity and it is controlled by temperature and emissivity. Hence, a stepwise method is required to estimate emissivity over a spectral region of interest. From each radiance spectrum measured, an equivalent temperature spectrum is determined by inversion of the radiance using Planck's equation. The stepwise 

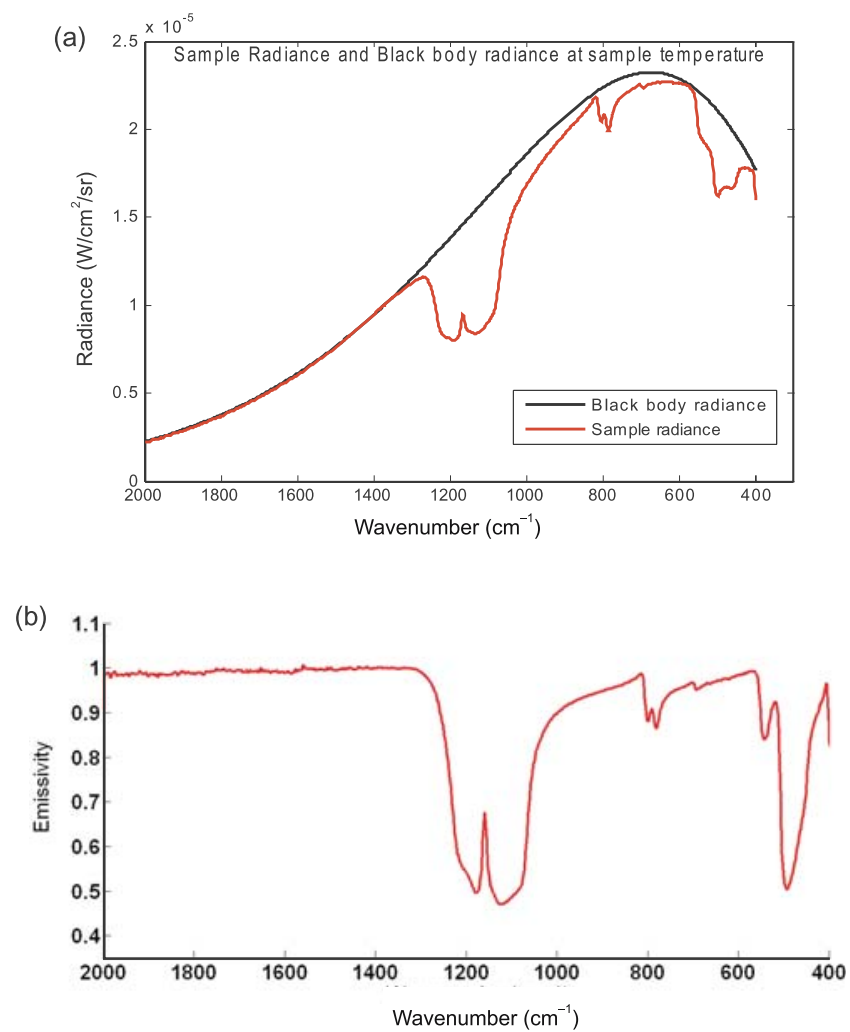

Figure 5. (a) Calibration technique to obtain emissivity of Quartz. The emissivity is obtained by dividing the sample radiance and blackbody radiance at same temperature, to generate the emissivity of a sample as shown in (b).

method used to derive emissivity from the measured quantity is described below.

The Planck's law describes the spectral radiance of electromagnetic radiation at all wavelengths from a blackbody at temperature $T$. As a function of frequency $\nu(\mathrm{Hz})$ can be expressed as:

$$
B(\nu, T)=\frac{2 h \nu^{3}}{c^{2}} \frac{1}{e^{h \nu / k T}-1} .
$$

This equation represents the emitted power per unit area of emitting surface, per unit solid angle, and per unit frequency, where $h$ is the Planck's constant, $c$ the speed of light and $k$ is Boltzmann constant.

The above equation as a function of wavelength $\lambda(\mu \mathrm{m})$ for unit solid angle is written as:

$$
B(\lambda, T)=\frac{2 h c^{2}}{\lambda^{5}} \frac{1}{e^{h c / \lambda k T}-1} .
$$

The third spectral unit, commonly used in spectroscopy, is wavenumber, the number of waves per $\mathrm{cm}$. The radiance in terms of Planck's equation as a function of appropriate wavenumber $\tilde{\nu}\left(\mathrm{cm}^{-1}\right)$ can be written as:

$$
B(\tilde{\nu}, T)=\frac{2 h c^{2} \tilde{\nu}^{3}}{e^{h c \tilde{\nu} / k T}-1}
$$

where $B(\tilde{\nu}, T)$ is the spectral radiance. With the use of above equation, temperature can be derived from radiance or radiance from temperature. The radiance data is converted to brightness temperature by inverting Planck's equation (4) (Ruff et al 1997),

$$
T=\frac{h \nu}{\operatorname{In}\left[2 h c^{2} \tilde{\nu}^{3}(B \tilde{\nu})^{-1}+1\right] k}
$$

In emission spectroscopy, the detector measures the variations in the voltage. The voltage varies as a function of wavelength and temperature of the sample or blackbody according to the following equation given by Christensen and Harrison (1993).

$$
\begin{aligned}
V_{\text {meas }}(\lambda, T)= & {\left[\varepsilon_{\text {samp }}(\lambda) B_{\text {samp }}(\lambda, T)\right.} \\
& +R_{\text {samp }}(\lambda) \varepsilon_{\text {env }}(\lambda) B_{\text {env }}(\lambda, T) \\
& \left.-\varepsilon_{\text {inst }}(\lambda) B_{\text {inst }}(\lambda, T)\right] F
\end{aligned}
$$

where $V_{\text {meas }}$ is the measured quantity, $\varepsilon_{\text {samp }}$ is the emissivity of the sample in the required quantity, the term $B$ is used to represent 'radiance' which depends on the temperature according to Planck equation. $B_{\text {samp }}$ is the radiance of sample that varies with wavelength and temperature according to Planck equation. $\varepsilon_{\text {inst }}(\lambda) B_{\text {inst }}$ is the instrument energy which includes all the energy that passes through the instrument and is not originating from the sample. $R_{\text {samp }}(\lambda) \varepsilon_{\text {env }}(\lambda) B_{\text {env }}(\lambda, T)$ is the energy term that is emitted by the environment and reflected off the sample towards the instrument. $F$ is the response function of the instrument which affects all of the above factors. The crucial part of emissivity measurement depends on how accurately the each, the above factors are taken care and accounted. The method related to the measurement of instrument response, energy, sample emissivity, etc., was developed by Ruff et al (1997), which is as follows.

\subsection{Instrument response function}

Response function represents the conversion factor between the detector output in volts and sample output in watts. Since all the components of the 


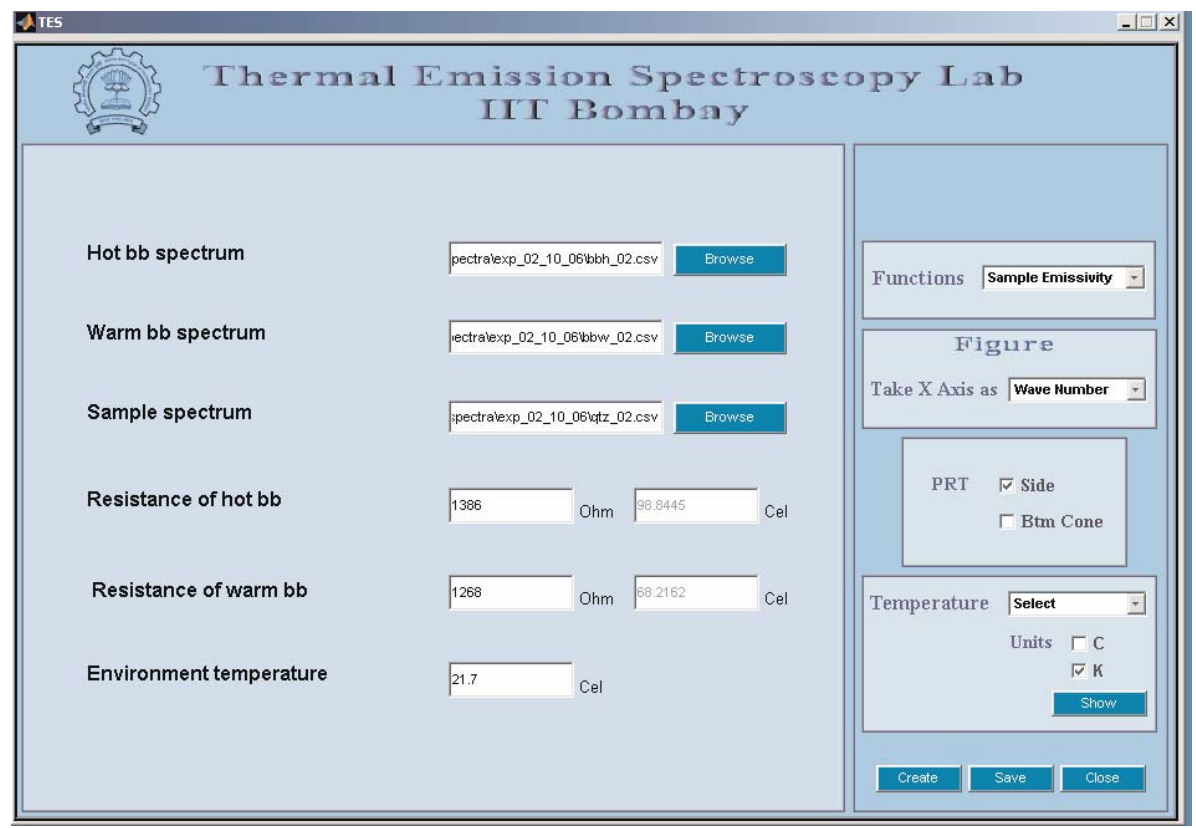

Figure 6. The calibration window developed in MATLAB as a user friendly window interface.

spectrometer do not respond perfectly at all wavelengths, it needs to be corrected. This correction is made by measuring a blackbody at two different known temperatures, and equation (4) is used to derive the radiance of the blackbody at known temperatures. A blackbody is an object that absorbs all electromagnetic radiation that falls onto it. In the case of a perfect blackbody, emissivity is one and reflectivity is zero. The equation (6) is hence reduced to equation (7).

$$
\text { Response function, } F=\frac{V_{b b}\left(T_{1}\right)-V_{b b}\left(T_{2}\right)}{B_{b b}\left(T_{1}\right)-B_{b b}\left(T_{2}\right)}
$$

where $V_{b b}$ is the voltages or energy measured for blackbody at temperatures of $100^{\circ} \mathrm{C}\left(T_{1}\right)$ and $70^{\circ} \mathrm{C}$ $\left(T_{2}\right) . B_{b b}$ is the blackbody radiance calculated using Planck's equation for known temperatures $T$. The instrument response function is a quantity that is independent of instrument energy and a small variation in temperature or any other component in the spectrometer should not have any significant effect on the response function. Hence, any variation in response function with time is not acceptable. The variation in the response function is therefore, monitored for over a period of time and is plotted in figure 7 .

\subsection{Instrument energy}

Instrument energy is the component of energy that does not come from the sample target. Using the

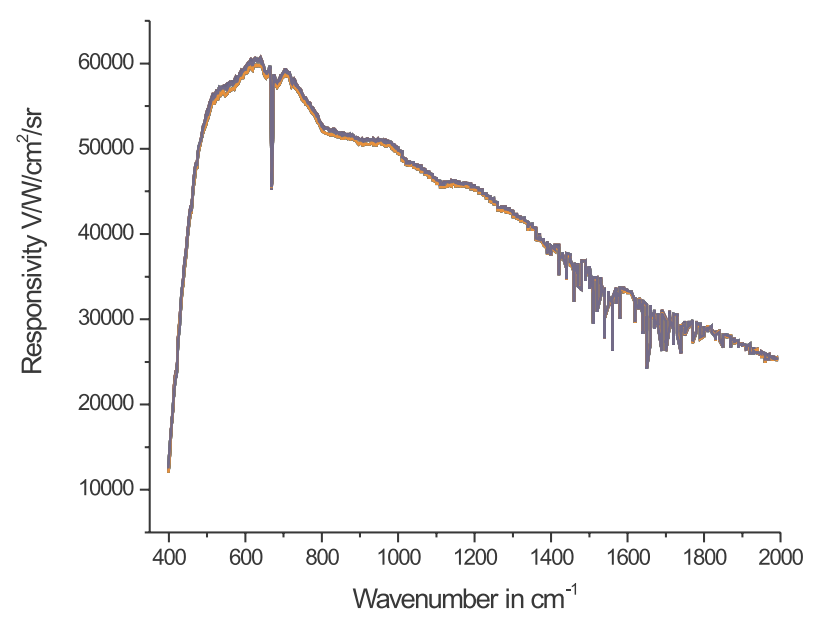

Figure 7. Instrument response function measured for 40 different times over two months. The standard deviation is less than $1 \%$.

instrument response function determined from the above equation, blackbody radiance for a particular temperature and voltage measured for that particular temperature instrument energy can be calculated using the following equation:

$$
\varepsilon_{\text {inst }} B_{\text {inst }}=B(T)-\frac{V_{\text {meas }}(T)}{F} .
$$

\subsection{Sample emissivity}

Emissivity $(\varepsilon)$ is derived as the ratio of calibrated sample radiance to that of sample radiance at $T$. Here, sample radiance is the radiance of a 
blackbody at sample temperature (calculated using Planck's equation for blackbody radiation) and calibrated sample radiance (emitted sample radiance) is the ratio of measured energy emitted from the sample $\left(V_{\text {samp }}\right)$ to instrument response function. Measured energy emitted from the sample $\left(V_{\text {samp }}\right)$ is the measured quantity available from the instrument after measuring the sample. Sample temperature is derived from calibrated sample radiance $\left(B_{\text {samp }}\right)$ by rearranging equation $(6)$ in the following format,

$$
\begin{gathered}
\varepsilon_{\text {samp }}(\lambda) B_{\text {samp }}(\lambda, T)+R_{\text {samp }}(\lambda) \varepsilon_{\text {env }}(\lambda) B_{\text {env }}(\lambda, T) \\
=\frac{V_{\text {samp }}}{F}+\varepsilon_{\text {inst }} B_{\text {inst }} .
\end{gathered}
$$

The above equation will get reduced into the following equation when $\varepsilon_{\text {samp }}=1$ and $R_{\text {samp }}=0$, an assumption made to isolate $B_{\text {samp }}$ so that the temperature can be calculated by putting $B_{\text {samp }}$ value in the Planck equation. The assumption is valid at the Christiansen frequency of the spectrum.

$$
B_{\text {samp }}(\lambda, T)=\frac{V_{\text {samp }}}{F}+\varepsilon_{\text {inst }} B_{\text {inst }} .
$$

Since the Christiansen frequency is not known prior, a brightness temperature is calculated for all wavelengths of the calibrated sample radiance using equation (5). Henry (1948) defined Christiansen frequency as the frequency at which the index of refraction $(n)$ of a sample becomes equal to that of the surrounding medium. The highest temperature is then used as the sample temperature. The sample temperature is then used in Planck equation for all wavelengths to derive the sample radiance $\left(B_{\mathrm{samp}}\right)$. Sample reflectivity $R_{\text {samp }}$ is converted in terms of emissivity using Kirchhoff's law relating reflectivity and emissivity $(R=1-\varepsilon)$. Substituting $\varepsilon$ for $R$ using Kirchhoff's law eliminates the term $R$ from the emissivity calculation (Ruff et al 1997). The emissivity of sample then becomes,

$$
\varepsilon_{\text {samp }}(\lambda, T)=\frac{\begin{array}{c}
\left(V_{\text {meas }}(\lambda, T) / F\right)-B_{\text {env }}(\lambda, T) \\
+B_{\text {inst }}(\lambda, T)
\end{array}}{B_{\text {samp }}(\lambda, T)-B_{\text {env }}(\lambda, T)},
$$

$\varepsilon_{\text {env }}$ and $\varepsilon_{\text {inst }}$ are eliminated from the equation as they are considered to be unity.

The calibration software is essential, as the final sample emissivity will be derived only after the above steps of calibration. Emissivity spectra from particulates $(700-1000 \mu \mathrm{m})$ of pure quartz are generated repeatedly to assess the reproducibility of the spectra. Figure 6(b) shows the reproduced spectra of quartz. The calibration program is coded in MATLAB 7.0. The program is developed with a front-end user friendly graphical interface for Windows Operating System (figure 6). The inputs required are $V_{b b}\left(T_{1}\right)$ (voltage measured for hot blackbody), $V_{b b}\left(T_{2}\right)$ (voltage measured for warm blackbody), $T_{1}$ (hot blackbody temperature), $T_{2}$ (warm blackbody temperature) and $V_{\text {sample }}$ (voltage measured for the heated sample). Instead of direct measurement of $T_{1}$ and $T_{2}$, two platinum resistors are placed inside the blackbody to measure the resistance shown at each temperature. The software converts the resistance into temperature $\left({ }^{\circ} \mathrm{C}\right.$ or $\left.\mathrm{K}\right)$. The other input required is the environmental temperature. The final output is the sample emissivity in the form of a graphical plot (figure 5b).

\section{Spectral deconvolution}

Deconvolution is an algorithm-based approach to reverse the effects of convolution or mixing. Deconvolution technique is used in signal processing to find the solution of a convolution equation of the form $\Sigma f x g=h$, where $h$ is the recorded signal, $f$ is the signal of individual component and $g$ is the function which transforms the individual component signal to the mixture signal. Spectral deconvolution is a data reduction technique that has been used for a variety of scientific problems that involves mixture analysis (Ramsey and Christensen 1998). Linear and non-linear deconvolution methods have been developed and studied by various workers for the VIS-NIR region (Sunshine et al 1990; Johnson et al 1992). The thermal infrared (TIR) portion of electromagnetic spectrum has been deconvolved using linear approach by various workers (Thomson and Salisbury 1993; Hamilton et al 1997; Ramsey and Christensen 1998). The simplest approach is the stochastic approach to calculate a modal analysis of rock from infrared spectrum that is based on the assumption that the spectrum of a mixture (rock) is a linear combination of the spectra of its components (minerals). The fundamental assumption of linearity in the TIR region is that the surface scattering is the predominant scattering process in TIR region compared to that of volume scattering, because of the higher absorption coefficient of minerals in this region. Previous workers have either concentrated on the VIS-NIR portion of the spectrum where reflection spectra show nonlinearity upon mixing (Clark 1983; Johnson et al 
1992) or with reflection spectra of TIR region (Thomson and Salisbury 1993) which is linear on mixing. Deconvolution modeling of TIR spectrum is based on linear mixing of end members. The linearity of TIR spectra was assumed from very early days (Lyon 1965).

The argument of the technique is that the emitted energy from the surface of multimineral rock surfaces is a combination of the energy radiated from each component in proportion to its aerial abundance percentage. For this linearity, the aerial percentage of surface-end member minerals with known particle sizes translate into volume abundance. If the spectra of pure-end member minerals are known, then any mixed spectra of rock sample can be deconvolved through a least squares linear fit (Ramsey and Christensen 1998). This would give percentage abundances of each inputend member minerals including their measures of modal quality. Therefore, because of linear mixing in the TIR region, the deconvolution provides a rapid means of assessing mineral assemblages of an exposed surface. Ramsey and Christensen (1998) determined that the deconvolution accuracy of TIR spectra is close to $94 \%$.

The deconvolution is achieved using linear least square equations. The algorithm developed for linear deconvolution can be written based on weighted average linear equation as follows:

$$
\varepsilon(\lambda)_{r}=a_{m 1} \varepsilon(\lambda)+a_{m 2} \varepsilon(\lambda)_{m 2}+\cdots+a_{m n} \varepsilon(\lambda)_{m n},
$$

where $\Sigma a_{m n} \sim 1$,

where $\varepsilon(\lambda)_{r}$ is the emissivity of rock which is a function of $\lambda$, the wavelength and $\varepsilon(\lambda)_{m n}$ represent the spectra of $n$th individual mineral component of the rock mixture which has a weightage of $a_{m n}$ or the per cent weightage of the individual mineral component which represents the aerial percentage of the individual mineral component.

At a specific wavelength $\lambda$, the emissivity $\varepsilon$ of a mixed spectrum of $n$ end members is equal to the aerial percentage $\omega$ of each end member times the emissivity with some residual error. The residual error is assumed to be the difference between the measured and modeled spectrum,

$$
\varepsilon(\lambda)=\sum \varepsilon(\lambda)_{i} \omega_{i}+\text { error }
$$

Several linear retrieval algorithms have been developed and modeled to determine the modal analysis of a rock spectrum (Adams et al 1986; Johnson et al 1992; Ramsey and Christensen 1998).
The present study is based on the model developed by Ramsey and Christensen (1998).

\subsection{Least square retrieval algorithm}

The mathematical expression of a rock spectrum is stated as:

$$
\varepsilon(\lambda)_{r}=\sum_{1}^{n} a_{m n} \varepsilon(\lambda)_{m n}+\text { error }
$$

$\sum a_{m n} \varepsilon(\lambda)_{m n}$ is the summation of emissivity of pure minerals at each wave length multiplied to the weight percentage of each mineral component. The weight percentage of each mineral component represents the proportion of each mineral contributing to the mixture spectra. Deconvolution is achieved using matrix algebra to solve a system of equations. Emissivity of a mixture is considered as a column matrix $B$ with $j$ number of rows and each row represents the emissivity of the mixture at wavelength $\lambda_{j}$ and there will be $j$ number of rows. Emissivity of pure minerals which depends on wavelength forms a matrix with $j$ number of rows and $n$ number of columns and each column is the emissivity of the representative mineral at wavelength $\lambda$. Thus the emissivity of $n$ number of end member minerals at wavelength $\lambda$ forms $n$ number of column of the matrix $A$. The weight percentage of each mineral component will form a column matrix with $n$ number of rows as is termed as matrix $X, A$ is a $j x n$ matrix. The matrix $A$ has $n$ mineral components with emissivity as the value that is dependent on the wavelength. Each column in matrix $A$ has $j$ rows and each row has a value which is the emissivity of the $n$th mineral component at wavelength $\lambda_{j} . X$ is $n x 1$ matrix where $n$ is the number of mineral components that contribute to the rock spectra. Hence, equation (14) can be rewritten in the form of matrix function and an algorithm is derived to deconvolve the mixture spectrum into its pure mineral components.

$$
A(j x n) X(n x 1)=B(j x 1) .
$$

In this algorithm, the available data are the emissivity of mixture for a range of wavelength denoted as $\lambda_{j}$ and the emissivity of the pure minerals used as the end members for a range of wavelengths same as that of the mixture spectra. The 'unknowns' in the above matrix function is the proportion of each mineral component in the mixture spectra. Therefore, equation (15) is rearranged to get the unknown matrix $X$. 


$$
X(n x 1)=\frac{B(j x 1)}{A(j x n)} .
$$

Equation (16) is rearranged into the following equation to generate the unknown matrix $X$ :

$$
X=\left(A^{T} A\right)^{-1} A^{T} B .
$$

Equation (17) is the matrix equation used to derive the unknown matrix $X$ which contains the weight percentage or the proportional abundance of each pure mineral component in the mixture spectra. After finding the $n$ number of unknown quantity the matrix $X$ is put back in the original equation (15) to derive the modeled spectrum of the mixture. The spectrum of the mixture derived experimentally is used to derive the unknown in the matrix equation (17). The error in the modeling is the amount of deviation of the modeled spectrum from the experimental spectrum. The error of the model is calculated using root mean square technique. The error is calculated as the RMS difference between the spectrum of the mixture generated experimentally to that of modeled (modeled spectrum is the summation of each mineral spectrum multiplied to its weight percentage).

The number of end-member minerals used for the algorithm is selected manually. An iterative method is adopted. The number of end members can be any number below the number of wavelength channels. In the present study, using the present FTIR instrument, the number of wavelength channels is around 830 with a spectral sampling of $4 \mathrm{~cm}^{-1}$. The measurement is from $2000 \mathrm{~cm}^{-1}$ to $400 \mathrm{~cm}^{-1}$ in wavenumber. Hence, the number of end members can be any number from 1 to 830. The actual end members present in the mixture will be giving a positive weightage value, while the absence of an end-member mineral chosen manually is indicated by a negative value. In the subsequent calculation, the end member with negative weightage value is omitted and the RMS value is calculated in each case. The process is repeated until the entire end members chosen are shown positive value with an RMS value approximately equal to zero, and until a visually best fitted spectrum is modeled.

\subsection{End-member minerals}

The accuracy of the deconvolution technique is dependent upon the end-member minerals with correct compositional and structural information. The Arizona State University mineral library contains (ASU spectral library) spectra of most of the rock forming minerals with detailed information on compositional purity and quality (Christensen et al 2000). Most library minerals are granular samples with sizes ranging from 700 to $1000 \mu \mathrm{m}$, as this size ranges can be used for the deconvolution of solid samples (Ramsey and Christensen 1998; Feely and Christensen 1999). The algorithm for deconvolution is coded using MATLAB and a user friendly graphical interface is made to do the deconvolution manually (figure $8 \mathrm{a}$ and $\mathrm{b}$ ). This algorithm requires three inputs:

- spectrum of the sample to be deconvolved,

- wavelength range over which to perform the linear least square fit, and

- library of pure mineral spectra (end members).

In the deconvolution window, the mineral spectral library of Arizona State University (ASU) is kept as separate window as ASU library (figure 8), while the new minerals being added including powder spectra are kept in a new window with an IITB code. Outputs from the algorithm includes, the weightage of each end member, modeled best fit spectrum, and root mean square RMS) error value, which is the average error over the entire spectral region. The RMS value is used as an indicator for the goodness of fit for all the iteration of measured spectra (Ramsey and Christensen 1998; Feely and Christensen 1999). Initially each spectrum is deconvolved choosing selected-end member libraries by looking at the spectral shape characteristics. As suggested by Christensen et al (2000), initially the iteration is to run using the entire library to find a best fit. In the present study, it is observed that often multiple iteration using group of minerals representing particular group or rocks are more advantageous than using the entire library initially. For an accurate modeling of the spectrum, the approximate range of composition present in the sample must be present as end members (Feely and Christensen 1999; Hamilton and Christensen 2000). Since even with robust mineral group of end-member mineral phases, it is unlikely that any end member will have an identical composition as that of the mineral in the sample (Hamilton et al 1997). Hamilton et al (1997) and Ramsey and Christensen (2000) demonstrated that lack of an exact match can be compensated for in the best fit model, if several compositionally related end members are also included in different proportions to essentially reduce the spectral contribution, of a phase in the measured spectrum.

Rock samples usually have lower spectral contrast than the library end-member minerals, since the spectral library uses spectra of particulate minerals of larger size fraction $(710-1000 \mu \mathrm{m})$, 


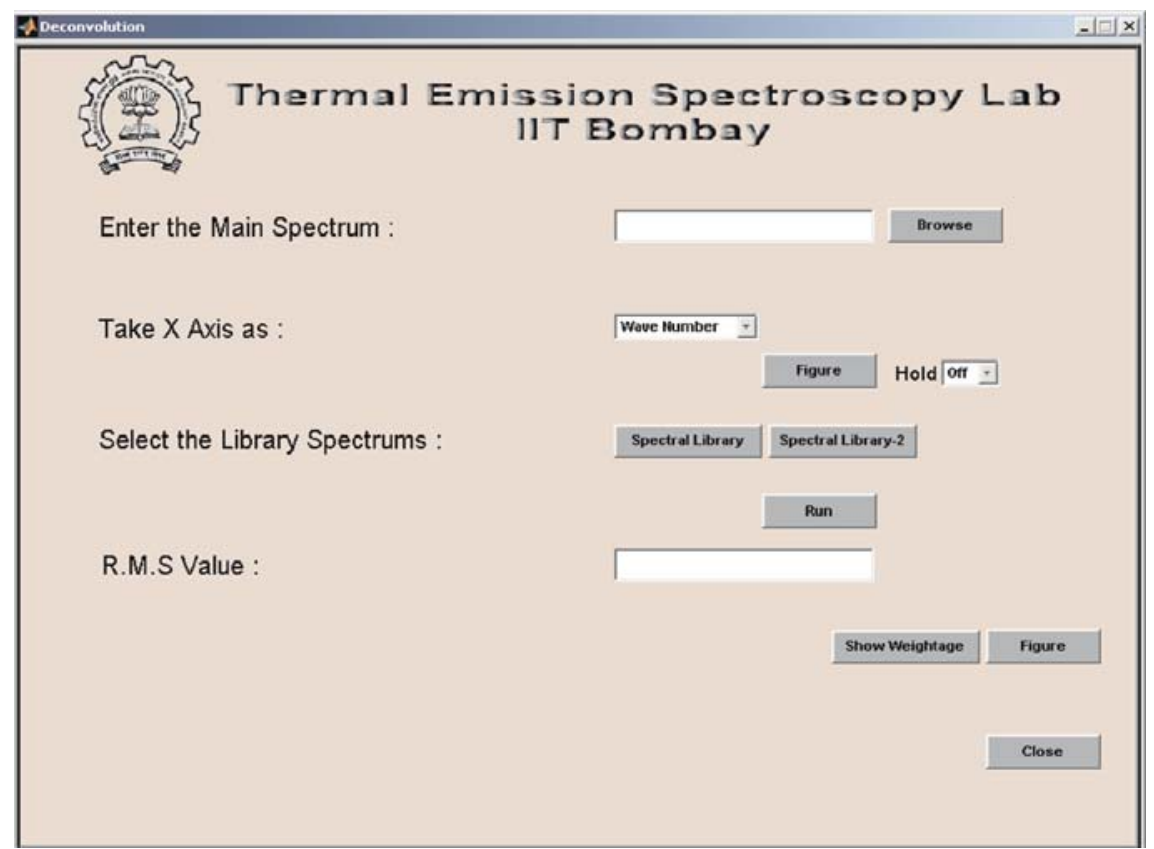

Figure 8. The deconvolution window developed in MATLAB as a window interface. The spectral library 1 represents ASU library, while the second represents the IITB library.

because they can be handpicked to reduce the contaminants, and due to the difficulty in getting large crystals of pure minerals. However, it was observed that decreasing the mean particle size of the sample, particularly greater than $65 \mu \mathrm{m}$ results in uniform decrease in the spectral contrast, without affecting the positions of absorption bands (Salibury and Wald 1992; Moersch and Christensen 1995; Ramsey and Christensen 1998). In such cases it is observed that when the measured rock spectrum is significantly shallower than the combined mineral components, the algorithm uses a featureless mineral spectrum to compensate it. To overcome this limitation, a blackbody spectrum, which has unit emissivity at all wavelengths, is added as one of the end members to the mineral library (Hamilton et al 1997, 2001; Feely and Christensen 1999).

As an example, we present here the thermal emission spectra of a granite and anorthosite and its deconvolved model mineral abundances of the rock spectra measured in the TES laboratory at IIT-Bombay (figure $9 \mathrm{a}$ and $\mathrm{b}$ ). The black coloured spectrum is the measured TIR rock spectrum, while red coloured spectrum is the modeled spectrum after manually selecting minerals from spectral library. The deconvolution algorithm models the mixture and calculates the difference in the measured and modeled spectra, and also weightage of each mineral that contribute the spectra. The difference in the modeled and measured spectra is expressed as RMS error.

\section{Lunar orbiter thermal emission spectrometer (LOTES)}

The thermal emission spectroscopy is a highly potential technique for remote sensing mapping of compositions on planetary surfaces. The mid-IR region between 4 and $25 \mu \mathrm{m}$ holds the key to estimate the mineralogical and bulk chemical composition of rocks and minerals. The TES technique was approved by NASA for the Mars mission during eighties, and successful mapping of Mars surface geology by the orbiters, viz., Mars Observer and THEMIS, and the rovers (Spirit and Opportunity) have further proved its worthiness, advocated earlier by Christensen et al (1985) for Mars composition mapping using the emission technique. However, with regard to Moon, the conclusions drawn based on the observations and results of several workers based on ground based observations, Goetz (1968); Murcray (1970); Potter and Morgan (1981); Tyler et al (1988); Lucey et al (1989) led to the view that emission spectroscopy or the mid-IR region of Moon is not a useful technique for lunar remote sensing. However, Lucey (1991) analysis of the same data of the above studies concluded that the earlier analysis suffered from serious signal-to-noise (SNR) and wavelength range limitations. This resulted in the spectral differences difficult to interpret in the mid-IR region. Later, Sprague et al (1992) based on scaled spectral emissivity data of five locations on the illuminated lunar surfaces showed existence of several 


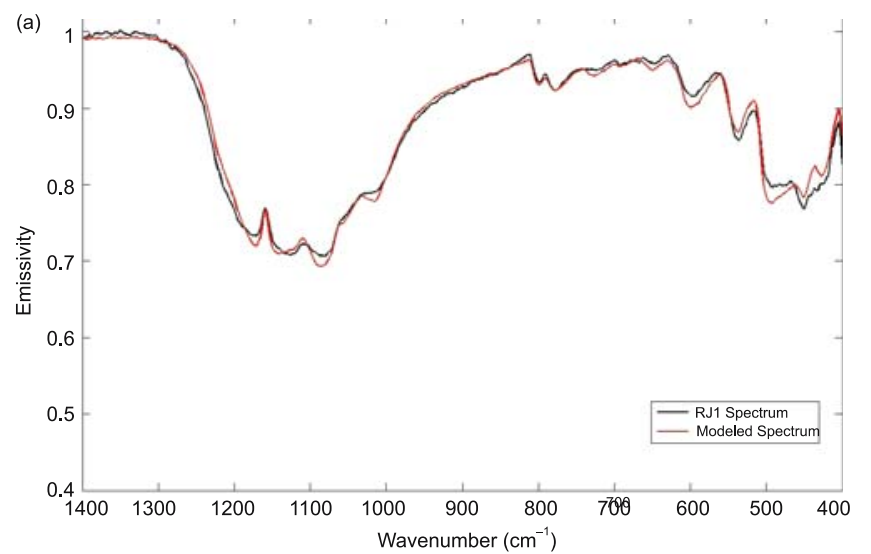

\begin{tabular}{|lcc|}
\hline Modeled minerals & $\begin{array}{c}\text { Model derived modes } \\
\text { (wt } \%)\end{array}$ & $\begin{array}{c}\text { Recalculated } \\
\text { (wt } \%)\end{array}$ \\
\hline Biotite BUR-840 & 5.06 & 5.08 \\
\hline Microcline BUR-3460 & 54.1 & 54.37 \\
\hline Quartz IITB & 33.9 & 34.08 \\
\hline Orthoclase IITB & 0.66 & 0.66 \\
\hline Labradorite IITB & 5.76 & 5.78 \\
\hline Blackbody & -0.16 & \\
\hline & & \\
RMS Error $: \mathbf{0 . 0 0 8}$ & & \\
\hline
\end{tabular}

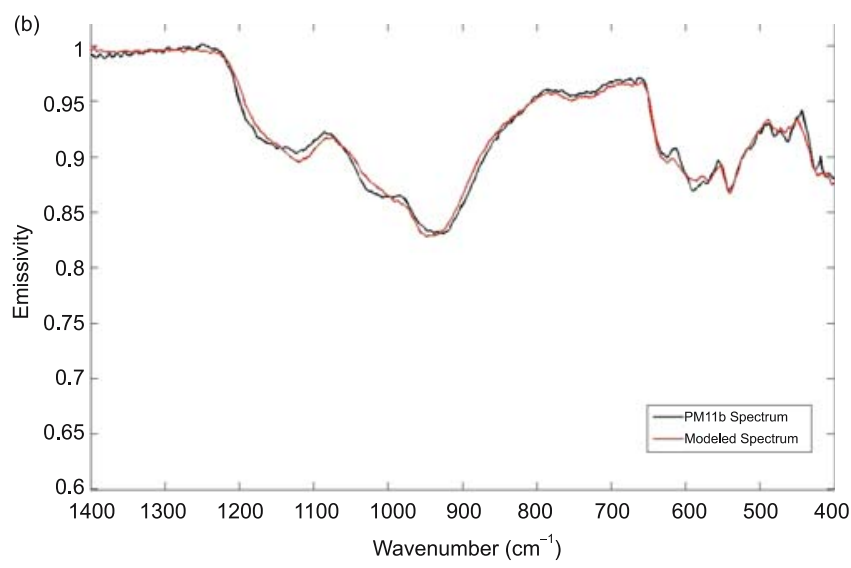

\begin{tabular}{|lcc|}
\hline Modeled minerals & $\begin{array}{c}\text { Model derived modes } \\
\text { (wt\%) }\end{array}$ & $\begin{array}{c}\text { Recalculated } \\
\text { (wt\%) }\end{array}$ \\
\hline Bronzite BUR-1920 & 0.80 & 0.62 \\
\hline Forsterite AZ-01 & 2.59 & 1.94 \\
\hline Augite HS-119.4B & 8.40 & 6.52 \\
\hline Anorthite WAR-5759 & 28.73 & 22.9 \\
\hline $\begin{array}{l}\text { Labradorite WAR- } \\
\text { RGAND01 }\end{array}$ & 88.39 & 68.61 \\
\hline Blackbody & -28.72 & \\
\hline RMS Error : $\mathbf{0 . 0 0 5}$ & & \\
\hline
\end{tabular}

Figure 9. Measured and modeled emissivity spectra a granite-RJ1 (a) and anorthosite-PM11b, and (b) its spectrally derived mineral abundance.

differences, which could be interpreted in terms of differences in their mineralogical compositions. Nash et al (1993) have discussed a comprehensive review on the evaluation of infrared emission spectroscopy for mapping Moon's surface from lunar orbit based on their experience of handling TES for Mars orbiter mission.

The TES would be useful to address some of the most significant lunar science issues, viz:
- types of feldspars and opaques,

- presence of silicate rocks,

- thermophysical properties of the lunar surface, and

- presence of volatile constituents in the poles (south Aitken basin).

Based on ground based telescopic observations and from the petrological and chemical studies of rock samples collected in the course of Apollo programme, and the soils returned by the robotic spacecraft of the USSR show that the surface of Moon predominantly consists of igneous rocks, mainly basalt and anorthosite (Mason and Melson 1970). The dominant mineral phases include olivine, pyroxenes, plagioclase and Fe-Ti minerals. However, studies by Marvin et al (1990) and Jollif (1990) show evidences of silicate rocks, granite and monozodorite. This clearly shows that the lunar surfaces might be more varied in their composition than thought based on the Apollo samples. Moreover, evidences of similar quartzofeldspathic rocks have recently been found on Mars (Bandfield et al 2004; Bandfield 2006). The exposures were clearly visible in Thermal Emission Imaging System (THEMIS) colour radiance images, and Thermal Emission Spectrometer (TES) surface emissivity data. Both the TES and THEMIS data indicate the presence of $8-9.5 \mu \mathrm{m}$ absorption band (Restrahalen band) compared to the surrounding basaltic plains.

It is therefore, important that global mapping of the Moon's surface needs to be carried out using a thermal emission spectrometer between 4 and $25 \mu \mathrm{m}$ for mapping evidences for varied lithologies. However, the difficulty on the Moon compared to Mars is the absence of atmosphere, which can introduce thermal gradients in the particulate materials due to loss of thermal convective heat transfer between grains. This can completely alter the spectral emittance of the sample surface, and result in increase in the spectral contrast (Logan et al 1973). Therefore, several laboratory emission measurements under vacuum conditions need be studied to understand the spectral behaviour. Of all the remote sensing based techniques, only thermal emission spectroscopy is the most sensitive technique for composition mapping. The spectroscopic techniques, VIS-NIR and mid-IR are sensitive to different parameters inherently related to the minerals (Farmer 1974). Abundance of quartz and alkali feldspars would be completely invisible to an NIR spectrometer, whereas the mid-IR spectrometer would not miss even the small abundance.

The updated version of the MERTIS instrument called the Selenological Radiometer and Thermal Infrared Imaging Spectrometer (SERTIS) has currently been proposed for the German 
Lunar Mission. Given the effectiveness of emission spectroscopy for composition mapping, and the new emission laboratory at IIT-Bombay, we propose that the next Indian Moon mission (Chandrayan-II) would consider a Lunar Orbiter Thermal Emission Spectrometer (LOTES) for a detailed mapping of the lunar surface.

\section{Acknowledgements}

The authors are grateful to the IIT-Bombay for their support. GM acknowledges funding provided by the ISRO-PLANEX for making the TES attachment. GM thanks Prof. P R Christensen and Dr Steve Ruff, ASU, US for their support and help in building the laboratory. He is extremely grateful to DST, Government of India for the BOYSCAST fellowship to visit ASU, US. GM and AN thank Mr. Rajeev Pillai for his help to make the calibration and deconvolution software in MATLAB. We thank the Associate Editor and the reviewer for their critical comments and suggestions.

\section{References}

Adams J B, Smith M O and Johnson P E 1986 Spectral mixture modeling: A new analysis of rock and soil types at the Viking Lander I site; J. Geophys. Res. 91 8098-8112.

Bandfield J L 2006 Extended surface exposures of granitoid compositions in Syrtis Major, Mars; Geophys. Res. Lett. 33 L06203, 1-4.

Bandfield J L, Hamilton V E, Christensen $\mathrm{P} R$ and McSween Jr H Y 2004 Identification of quartzofeldspathic materials on Mars; J. Geophys. Res. 109 E10009, $1-14$.

Christensen P R, Kieffer H and Chase S 1985 Determination of Martian surface composition by thermal infrared spectral observations; Lunar. Planet. Sci. XVI 125-126.

Christensen P R, Bandfield J L, Hamilton V E, Howard D A, Lane M D, Piatek J L, Ruff S W and Stefanov W L 2000 A thermal emission spectral library of rock-forming minerals; J. Geophys. Res. 105 9735-9739.

Christensen P R, Bandfield J L, Hamilton V E, Ruff S W, Kieffer H H, Titus T N, Malin M C, Moris R V, Lane M D, Clark R L, Jakosky B M, Mellon M T, Pearl J C, Conrath B J, Smith M D, Clancy R T, Kuzmin R O, Rousch T, Mehall G L, Gorelick N, Bender K, Murray K, Dason S, Greence E, Silverman S and Greenfield M 2001 Mars global surveyor thermal emission spectrometer experiment: Investigation description and surface science results; J. Geophys. Res. 106(E10) 23,823-23,872.

Christensen P R, Bandfield J L, Bell J F, Gorelick N, Hamilton V E, Ivanov V E, Jakosky B M, Kiefer H H, Lane M D, Malin M C, McConnochie T, McEwen H Y, Mehall G L, Moersch J E, Nealson K H, Rice J W, Richardson M I, Ruff S, Smith M D, Titus T N and Wyatt M B 2003 Morphology and composition of the surface of Mars: Mars Odyssey THEMIS results; Science 300 2056-2061.
Christensen P R and Harrison T S 1993 Thermal infrared emission spectroscopy of natural surfaces: Application to desert varnish coatings on rocks; J. Geophys. Res. 98 19,819-19,834.

Clark R N 1983 Spectral properties of mixtures of montmorillonite and dark carbon grains: Implications for remote sensing minerals containing chemically and physically adsorbed water; J. Geopys. Res. 88 10,635-10,644.

Clark R N 2004 Spectroscopy of rocks and minerals, and principles of spectroscopy; In: Molecules to Planets: Infrared Spectroscopy in Geochemistry, Exploration Geochemistry and Remote Sensing (eds) King P L, Ramsey M S and Swayze G, Mineral. Assoc. Canada, Short Course 33 17-50.

Farmer V C 1974 The infra-red spectra of minerals; Mineral. Soc. London, $539 \mathrm{p}$.

Feely K C and Christensen P R 1999 Quantitative compositional analysis using thermal emission spectroscopy: Application to igneous and metamorphic rocks; J. Geophys. Res. 104 24,195-24,210.

Formisano V, Angrilli F, Arnold G, Atreya S, Bianchini G, Biondi D, Blanco A, Blecka M I, Coradini A, Colangeli L, Ekonomov A, Esposito F, Fonti S, Giuranna M, Grassi D, Gnedykh V, Grigoriev A, Hansen G, Hirsh H, Khatuntsev I, Kiselev A, Ignatiev N, Jurewicz A, Lellouch E, Lopez Moreno J, Marten A, Mattana A, Maturilli A, Mencarelli E, Michalska M, Moroz V, Moshkin B, Nespoli F, Nikolsky Y, Orfei R, Orleanski P, Orofino V, Palomba E, Patsaev D, Piccioni G, Rataj M, Rodrigo R, Rodriguez J, Rossi M, Saggin B, Titov D and Zasova L 2005 The Planetary Fourier Spectrometer (PFS) onboard the European Mars Express mission; Planet. Space Sci. 53(10) 963-974.

Gillespie A R, Matsunaga T, Rokugawa S and Hook S J 1998 Temperature and emissivity from Advanced Spaceborne Thermal Emission and Reflection Radiometer (ASTER) images; IEEE Tran. Geosci. Remote Sen. 36 1113-1126.

Goetz A F H 1968 Differential infrared lunar emission spectroscopy; J. Geophys. Res. 73 1455-1466.

Hamilton V E and Christensen P R 2000 Determining the modal mineralogy of mafic and ultramafic rocks using thermal emission spectroscopy; J. Geophys. Res. 105 9717-9733.

Hamilton V E, Christensen P R and McSween H Y 1997 Determination of Martian meteorite lithologies and mineralogies using vibrational spectroscopy; J. Geophys. Res. $10225,593-25,603$.

Hamilton V E, Wyatt M B, McSween H Y and Christensen P R 2001 Analysis of terrestrial and Martian volcanic compositions using thermal emission spectroscopy: 2. Application of Martian surface spectra from the Mars Global Surveyor Thermal Emission Spectrometer; J. Geophys. Res. 106 14,733-14,746.

Hawthorne F C 1988 Spectroscopic methods in Mineralogy and Geology; Rev. Mineral. 18. Min. Soc. America.

Helbert J, Jessberger E, Benkhoff J, Arnold G, Banaszkiewicz M, Bischoff A, Blecka M, Calcutt S, Colangeli L, Coradini A, Erard S, Fonti S, Killen R, Knollenberg J, Kührt E, Mann I, Mall U, Moroz L, Peter G, Rataj M, Robinson M S, Spohn T, Sprague A, Stöffler D, Taylor F and Warrell J 2005 MERTIS A thermal infrared imaging spectrometer for the BepiColombo Mission; In: 36th Ann. Lun. Planet. Sci. Conf. 1753.

Henry R L 1948 The transmission of powder films in the infrared; J. Opt. Soc. Am. 38 775-789.

Jollif B L 1990 Fragments of quartz monzodiorite and felsite in Apollo 14 soil particles; Lunar Planet. Sci. XXI $571-572$ 
Johnson P M, Smith M O and Adams J B 1992 Simple Algorithms for remote determination of mineral abundances and particle sizes from reflectance spectra; J. Geophys. Res. 97 2649-2657.

King P L, Ramsey M S, McMillan P F and Swayze G 2004 Laboratory fourier transform infrared spectroscopy methods for geologic samples: Infrared Spectroscopy in Geochemistry, Exploration, and Remote Sensing (eds) King P, Ramsey M and Swayze G, Mineral. Assoc. of Canada, London 33 57-91.

Logan L M, Hunt G, Salisbury J and Balsamo 1973 Compositional implications of Christensen frequency maximums for remote sensing applications; J. Geophys. Res. 78 4983-5003.

Lucey P G 1991 Comparison of thermal emission spectroscopic measurements of the lunar surface: 1968-1990; Proc. Lunar. Planet. Sci. Conf. 21 417-423.

Lucey P B, Hawke B R and Bruno B 1989 Thermal infrared spectroscopy of the Moon; Bull. Am. Astron. Soc. 21970.

Lyon R J P 1965 Analysis of rocks by spectral infrared emission; Econ. Geology 60 715-736.

Marvin V B, Holmberg B and Lindstrom 1990 New pieces of the lunar granite-quartz monzodiorite puzzle; Lunar. Planet. Sci. XXI 738-739.

Mason B and Melson W G 1970 The Lunar Rocks; Wiley Interscience 179.

Maturilli A, Helbert J and Moroz L 2008 The Berlin emissivity database (BED); Planet. Space. Sci. 26 420-425.

Moersch J E and Christensen P R 1995 Thermal emission from particulate surfaces: A comparison of scattering models with measured spectra; J. Geophys. Res. 100 $7465-7477$.

Murcray F H D 1970 Infrared emissivity of lunar surface features, 1, balloone-borne observations; J. Geophys. Res. $752662-2669$.

Nash D B, Salisbury J W, Connel J E, Lucey P G and Christensen P R 1993 Evaluation of infrared emission spectroscopy for mapping the Moon's surface composition from lunar orbit; J. Geophys. Res. 98 23,535-23,552.

Nicodemus F E 1965 Directional reflectance and emissivity of an opaque surface; Appl. Opt. 4 767-773.

Potter A E and Morgan T H 1981 Observations of silicate reststrahlen bands in lunar infrared spectra; Proc. Lunar. Planet. Sci. Conf. 12B 703-713.

Ramsey M S and Christensen P R 1998 Mineral abundance determination: Quantitative deconvolution of thermal emission spectra; J. Geophys. Res. 103 $577-596$.
Ramsey M S 2004 Quantitative geological surface processes extracted from infrared spectroscopy and remote sensing; In: Infrared Spectroscopy in Geochemistry, Exploration Geochemistry and Remote Sensing (eds) King P L, Ramsey M S and Swayze G, Mineral. Assoc. Canada 33 197-224.

Ruff S W, Christensen P R, Barbara P W and Anderson D L 1997 Quantitative thermal emission spectroscopy of minerals: A laboratory technique for measurement and calibration; J. Geophys. Res. 102 14,899-14,913.

Salisbury J W and Wald A 1992 The role of volume scattering in reducing spectral contrast of reststrahlen bands in spectra of powdered minerals; Icarus 96 $121-128$

Salisbury J W, Wald A and D'Aria D M 1994 Thermal infrared remote and Kirchoff's law, 1. Laboratory measurements; J. Geophys. Res. 99 11,897-11,911.

Salisbury J W 1993 Mid-infrared spectroscopy: Laboratory data; In: Remote Geochemical Analysis: Elemental and Mineralogical Composition (eds) Pieters C M and Englert P A J (Cambridge: Cambridge University Press) 79-98.

Salisbury J W, Walter L S, Vergo Norma and Dana M D'Aria 1993 Infrared spectra of minerals; John Hopkins University Press, 280.

Sprague A L, Witteborn F, Kozloski R, Cruikshank D, Bartholomev M and Graps A 1992 The Moon: Midinfrared $(7.5-11.4 \mu \mathrm{m})$ spectroscopy of selected regions; Icarus 100 73-84.

Squyres S W, Arvidson R E, Ruff S, Geller R, Morris R V, Ming D W, Crumpler L, Farmer J D, Des Marais D J, Yen A, McLennan S, McCalvin M, Calvin W, Bell J F, Clark B C, Wang A, McCoy T J, Schmidt M E and De Souza P A 2008 Detection of silica rich deposits in Mars; Science 320 1063-1067.

Sunshine J M, Pieters C M and Pratt S R 1990 Deconvolution of mineral absorption bands: An improved approach; J. Geophys. Res. 95 6955-6966.

Thomson J L and Salisbury J W 1993 The mid-infrared reflectance of mineral mixtures $(7-14 \mu \mathrm{m}) ;$ Rem. Sen. Environ. 45 1-13.

Tyler A L, Kozlowski W and Lebosfsky L 1988 Determination of rock type of Mercury and Moon through remote sensing in the thermal infrared; Geophys. Res. Lett. 15 808-811.

Walter L S and Salisbury J W 1989 Spectral chracterisation of igneous rocks in the 8 to 12 micrometer region; J. Geophys. Res. 94(B7) 9203-9213. 